pear in the spectrum. This was clearly observed in Y. Lee's laboratory where a detailed investigation of the role played by both fluence and intensity at very high levels of excitation was performed on several molecules le.g. $\mathrm{SF}_{6}, \mathrm{CF}_{3} \mathrm{Cl}, \mathrm{N}_{2}$ $\mathrm{N}_{4}$ ) having different densities of states close to the continuum region ${ }^{3}$ ). In some cases (e.g. $\mathrm{C}_{2} \mathrm{~F}_{5} \mathrm{Cl}$ ) the average level of excitation can overcome more than one dissociation channel, so that the molecule can dissociate into different products. Experiments where the angular distribution and translational energy of the fragments were determined, have provided a check on the validity of statistical theories in the continuum, i.e. to study the redistribution of energy occurring there under unimolecular dissociation, the fragmentation process and the energy retained in each fragment. The main results are:

dissociation yield depends only on laser fluence, but the average level of excitation reached still depends on laser intensity; a large molecule is easily dissociated and acquires more energy then a small molecule at the same laser fluence. Most of the excess energy is retained as excitation energy in the fragments.

These conclusions seem to confirm the validity of the statistical theory describing IR multiphoton dissociation of most of the polyatomic molecules excited by conventional $\mathrm{CO}_{2}$ lasers $(\cong 100 \mathrm{~ns}$ pulse duration). The dependence on intensity of the average level of excitation reached above the dissociation threshold has left some hope for a practicable bond-selective photochemistry in the future, provided that very short (10-100 ps) and intense IR laser sources become available.

\section{BIBLIOGRAPHY}

1. For extended recent reviews of laser induced processes in molecules see:

(a) Letokhov V.S., Non Linear Laser Chemistry, Springer Series in Chemical Physics 22 (Springer Verlag) 1983;

(b) Laser Applications in Chemistry, Eds. G. Scoles and J. Wanner (Plenum Press, N.Y.) 1983, 151-160 and references therein;

(c) Laser Spectroscopy VI, Eds. H.P. Weber and W. Luthy (Springer Verlag, Heidelberg) 1983.

2. For further reading on molecular beams see:

Anderson J.B., Andres P.R. and Fenn B.J., Advances in Chemical Physics 10 (1966) 275-317.

3. Lee Y.T. and Shen Y.R., Phys. Today 33 (1980) 52.

4. Alimpiev S.S., Karlov N.V., Nikiforov S.M., Prokhorov A.M., Sertakov B.G., Khokhlov E.M. and Shtarkov A.L., Sov. J. Quantum Electron 13 (1983) 331.

\title{
Theory of Energetic Ion-Atom Collisions
}

\author{
J.S. Briggs, Freiburg, and K. Taulbjerg, Aarhus
}

(Universities of Freiburg and Aarhus respectively)

The theory of ion-atom collisions concerns the calculation of the atomic states resulting from the collision between two nuclei and their attendant electrons. Although the existence of nuclear resonances occasionally may play an interesting role, it is usually adequate, at the energies which are relevant to atomic physics, to consider all particles to interact only via the electromagnetic interaction and the nuclei to remain intact throughout the collision. The Hamiltonian governing the motion of the interacting atoms is known, and it is only a matter of solving the time-dependent Schrödinger equation or the equivalent equations of the time-independent formulation of scattering theory to calculate the probabilities of transition into the various excitation, break-up, or rearrangement channels which are energetically available.

From this point of view, it is quite natural to question the pertinence of further effort in this field. Most collision experiments in nuclear and particle physics have the ultimate aim of deducing the forces between the constituent particles. What then is to be learned from atomic-collision experiments? After all, there is not much need to test the validity of the time-dependent Schrödinger equation for nonrelativistic motion.

Nevertheless, aside from the enormous technological and astrophysical applications, the field of atomic collisions is of considerable fundamental interest. It is precisely the exact knowledge of the forces of interaction that allows a sensitive test of collision theory to be made, a possibility not so easily open to nuclear and particle theorists. Any theory of such collisions must rely on approximation procedures or model assumptions. Because of this, it is essential that the atomic interaction forces are known exactly so that the modelling of specific collision-dynamic features may be exposed to a unique test.

At first sight, it may seem that atomiccollision theory is simplified by the simple mathematical form of the static Coulomb interaction, which for most purposes can be considered to be dominant. Unfortunately, the infinite range and other peculiarities of the Coulomb force endow the theory of atomic colli- sions with a fundamental delicacy and invalidate most theorems and rigorous formulations applicable to short-range forces. Indeed, the standard methods of scattering theory are not applicable unless the interaction amongst asymptotic channels decays faster than the Coulomb interaction. Much effort is still devoted to the resolution of this fundamental problem, but a complete clarification has not yet emerged.

The problem is usually circumvented by arguing that it is acceptable to assume that Coulomb interactions are cut off or appropriately screened at distances extremely large on the atomic scale. After all, particle detectors are not set up in the laboratory infinitely separated from the scattering chamber. Then it is physically sound to expect that the description of specific processes occurring on the atomic scale will be independent of the details of the screening procedure if the screening radius is made sufficiently large. There is no evidence that this supposition is invalid, but it is essential to exert particular care to ensure complete consistency if difficulties with irregularities in the limit of a pure unscreened Coulomb force are to be avoided. Accordingly, we believe that ordinary formal scattering theory may be applied in atomic collisions if one is sufficiently careful, as seen in Panel 1.

The effect of an atomic collision is either to excite one or more electrons of either target or projectile to bound states, to transfer electrons from bound states around one nucleus to bound states around the other (electron capture), or to eject electrons into the continuum (ionization). Electron excitation and electron capture are uniquely defined as single-channel and rearrangement-channel processes, respectively. The situation is not so clear in the case of ionization. In principle, ionization is a break-up process, but it is convenient to talk about target ionization if the ejected electron is relatively slow with respect to the residual target ion and about capture to the projectile continuum if the ejected electron is slow with respect to the projectile. This distinction merely reflects the consideration that target ionization may be understood in a singlechannel approach, while capture to continuum requires a rearrangement-chan- 
Panel 1

It is a recurring theme of the theory of collisions of few-body systems to attempt to unravel the totality of interactions in terms of the separate interactions of pairs of particles. The mathematical expression of this is found, for example, in the Faddeev approach to many-body scattering theory. The aim in nuclear physics is to determine the separate two-body $T$ matrices and thereby the nuclear forces. In atomic-collision theory, a similar procedure is employed but with the aim of separately identifying those features of the collision arising from the nucleus-nucleus, electron-nucleus, or electron-electron interactions. Although mediated by the same force, the strengths and associated kinematics of these interactions are sufficiently different to make such a separation meaningful.

One such example of the dominance of one pair-wise interaction during the collision is provided by the impact of a highly charged ion or bare nucleus upon a hydrogen atom. The potential that binds the electron to the target is then so much weaker than the potential due to the incident projectile that it is reasonable to ignore the target potential during the encounter. From this point of view, the bound-electron state may be considered to be a wave packet incident on the pro- jectile, and the result of the encounter can accordingly be represented by the corresponding packet of scattered waves. However, it should be noted that the present wave packets, describing states of sharply defined energy, are not quite identical to the free wave packets employed in potential-scattering theory.

The momentum components of a free wave packet scatter independently under conservation of energy, i.e., on the energy shell. A bound-state wave packet naturally also scatters under conservation of energy, but that in turn implies that its individual momentum components scatter off the energy shell, the departure from the energy shell becoming small at sufficiently high velocities. The off-shell effect is ignored in what conventionally is referred to as the impulse approximation.

The condition for its validity is readily met with short-range potentials but becomes increasingly difficult to satisfy as the range is expanded. A proper treatment of atomic collisions therefore generally requires that off-shell effects be consistently incorporated. The generalized approach obtained when such offshell effects are retained in the impulse approximation is now commonly referred to as the Strong-Potential approximation. vant portion of Hilbert space probed by the collision. The former method is considered appropriate at high velocity and the latter at low velocity.

The theory for direct processes, i.e., transitions to excited or ionized target states, is well developed, and results are generally in good accord with experimental findings for total collision crosssections. Currently, the theoretical effort is devoted primarily to refined effects such as the influence of nuclear resonances, of structured projectiles, and of electron correlations. Indeed, one of the major remaining problems in a general atom-atom collision is the unravelling of those effects due to electron correlations existing in bound states before and after the collision, from those due to the correlated motion of electrons during the collision. A similar problem involving nuclear correlations exists in nuclear physics, and the theoretical methods employed in both fields are often not dissimilar (see Panel 2).

Perturbation theories are particularly useful at intermediate and high collision energies. Coupled channels methods become relevant at intermediate-to-low velocities, but it is well established that a fairly sizable basis is needed to represent important intermediate states during the collision. As the velocity is further reduced, charge-cloud oscillations between near-resonant levels begin to be significant, and one enters the molecular regime discussed below. The conclusion is, that the first Born approximation is generally invalid for electron capture. nel approach. Clearly, there must exist a region where the ejected electron interacts mutually with the projectile and the residual target atom.

This problem, i.e. three closely interacting unbound Coulomb particles, still poses a considerable challenge to theorists. In ion-atom collisions, the problem is that of one electron and two nuclei (or atomic cores), but the problem has much wider application. For example, the unbound motion of one nucleus and two electrons, as occurs in threshold ionization of atoms by electron or positron impact, is essentially the same problem. As effects in Coulomb fields generally extrapolate below threshold, the same three-body states occur in the highly correlated electronic motion found in auto-ionizing, doubly excited states of atoms. Similar considerations apply to the break-up of molecular fragments where the interaction is through long-range Coulomb forces.

Depending upon the collision velocity, explicit calculations on ion-atom collisions are normally done either in a perturbation or in a coupled channels approach. In the former, a limited number of interaction events is allowed (depending on the order of the perturbation theory) but in principle between an infinity of states, i.e., the complete Hilbert space of the final-arrangement channels. In the latter, an infinite number of interaction events is allowed (the dynamic equation is solved exactly) but between a limited number of states, hopefully chosen judiciously to span the rele-

\section{CHAIR OF LASER PHYSICS}

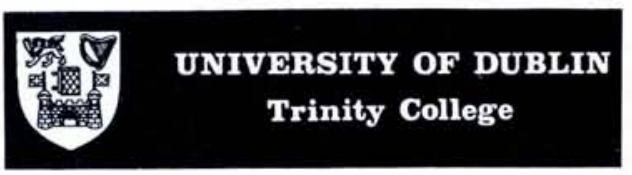

Applications are invited for this senior professorial appointment within the Department of Pure and Applied Physics. The duties associated with the post will include leadership of the Department's laser physics group, founded by Professor D.J. Bradley, FRS. The group is engaged in advanced research projects in nonlinear optics, semiconductor devices, and interdisciplinary studies, in well-equipped laboratories. It has attracted substantial research grants and contracts.

Candidates should have an established record of research in laser physics or nonlinear optics, together with ability to direct a substantial research effort and to contribute to the teaching of the Department.

Further particulars may be obtained from:
Telephone: 7729 41, exts 1722, 1123

\author{
G. H. H. Giltrap \\ Secretary to the College \\ Trinity College \\ Dublin 2, Ireland
}

to whom formal application should be made, preferably not later than 15 February, 1985. 


\section{Panel 2}

The basic kinematics of the direct ionization process in fast collisions may be understood in terms of a binary encounter of the active electron with the projectile. The single-collision nature of the direct ionization process is theoretically reflected in the fact that the first Born approximation is often excellent. On the other hand, the rearrangement process is not kinematically compatible with a single binary-encounter description; a free electron cannot be picked up in a single collision with an incident nucleus. In order to balance simultaneously the energy and momentum accounts, a third body is needed. Capture can occur in a single three-body collision or in a minimum of two separate binary collisions. That the former relies on the binding of the electron to the target nucleus whilst

It is experimentally impossible to distinguish between single- and multiplescattering contributions. The various terms naturally add coherently to the total transition amplitude, and it is in principle impossible to isolate a particular term unless that particular term for some reason gives a dominant contribution to the amplitude. In fact, the theoretical total capture cross-section is dominated by the double-scattering term in the high-velocity limit. However, the cross-section drops off so rapidly in this region that it is hardly measurable. Fortunately it is possible to isolate the Thomas double-scattering term in the differential capture cross-section at much lower velocities. Here, the dominant part of the cross-section is extremely forward-directed, while the double-scattering term primarily contributes around a definite angle determined by kinematical considerations. The existence of the 'Thomas peak' in the differential cross-section at this angle has been verified only in recent experiments. Much effort is currently devoted to a detailed test of the various theoretical formulations and approximation methods against this beautiful experimental demonstration.

An interesting aspect of the multiple scattering character of the electroncapture process is that multi-electron effects may be expected to enter at the same level. As an example, the Thomas double-scattering mechanism works equally well if the second collision is considered to take place with another target electron instead of the target nucleus. A kinematic consideration shows that capture at high velocities in this case is accompanied by the ejection of a fast electron perpendicular to the direction of incidence. the latter mechanism can occur with free electrons was recognized by Thomas, as early as in 1927, who gave a classical treatment of electron capture. Surprisingly, the fact that the three-body mechanism is described by the first Born approximation, but the double binary collision occurs only in the second Born approximation, was completely overlooked during the next thirty years. Even more surprisingly, the second Born approximation is now known to dominate over the first at high collision velocities. Indeed it is now generally accepted that singlecollision theories such as the first Born approximation are fundamentally unjustified for atomic rearrangement processes. The situation is best clarified in the case of asymmetric collisions such as protons on hydrogen-like heavy ions.

At lower velocities, other types of electronic interactions may contribute to the capture process, the significance of which is only beginning to be appreciated both theoretically and experimentally. Electron correlations present in the initial state may contribute to capture directly and certainly play a role in the unexpectedly frequent occurrence of multiple-electron capture. Recent evidence has appeared for the temporary existence of highly correlated, doubly excited electronic states during ionatom collisions. Such states have long been known in the resonant scattering of single electrons by atoms.

At collision velocities much smaller than relevant electron orbital velocities, the processes of electron excitation and capture become equally probable. This is because a collision complex is formed a temporary 'molecule', where the electrons lose memory of the nucleus to which they were originally attached. The slowness of the collision, however, in no way implies restrictions on the incident energy. The inner-shell electrons of very heavy ions move so quickly that incident-ion velocities corresponding to energies of $1 \mathrm{GeV}$ and above may still be slow on this scale.

The idea of the formation of a molecular complex during an ion-atom encounter, although a very old one, has had an enormous impact on the recent development of the theory of slow collisions. It is remarkable in its universality; the same type of processes occurring in protonhydrogen collisions at $25 \mathrm{keV}$ occur for the inner-shell electrons in a uraniumuranium collision at around $1 \mathrm{GeV}$. Unlike the situation in nuclear physics, the ion-atom collision complex has a well defined axis of symmetry, the internuclear axis. Indeed it is precisely the speed of contraction and rotation of this axis during the collision that decides the final distribution of the electron cloud.

The molecular model has the further advantage that the necessary ingredients of the theory, the diatomic molecular eigenfunctions for fixed internuclear axis, can be obtained directly from existing computer codes developed for use in quantum chemistry. However, the necessity to consider highly relativistic electrons, as in the collision of two very heavy atoms, has provided impetus for the development of programs which approximately solve the two-centre many-electron Dirac equation, a formidable task in itself.

It is under the influence of technological advances in the production of highly charged slow ions and the acceleration of the heaviest ions that new demands are being made upon the theory of atomic collisions. Slow ions with charges up to fifty are now attainable. Such ions readily capture electrons from neutral target atoms, and elementary considerations of the molecular-collision complex show that capture occurs predominantly into states with principal quantum number of the order of the ionic charge, that is, a highly inverted beam population is produced. The calculation of the precise distribution of electrons amongst the enormous number of available levels of different symmetry is a matter of some delicacy. Not only that, for projectiles of such high charge, the capture of many electrons may be the rule rather than the exception, and theory is only beginning to contemplate the dynamics of such mass motion of atomic electrons.

Theory using the molecular model has been able to explain the surprisingly high degree of inner-shell ionization occurring in collisions between heavy ions. For the heaviest ions in close collisions, the binding energy of such hole states may temporarily exceed twice the electron rest mass, opening up the possibility of pair creation. This has required the extension of the theory of atomic collisions to include direct coupling to the positron continuum.

The trend continues toward the acceleration of ions of increasingly higher charge to increasingly higher energies. Highly charged ions, even at relativistic velocities, can never be considered as a perturbation, and a future task for atomic theory is the development of a reliable, fully relativistic many-body scattering theory for aggregates of electrons and nuclei of high charge and momentum interacting purely through electromagnetic forces. 